Markus Klammer

\title{
Ornamente transzendental und empirisch. Zu Kants Kritik der Urteilskraft und zu den Tätowierungen der »Neuseeländer»
}

I.

In seiner Besprechung zweier jüngerer, großangelegter englischsprachiger Monographien zum Thema des Ornaments - James Trillings Ornament. A modern perspective (2003) und David Bretts Rethinking decoration. Pleasure and ideology in the visual arts (2005) - konstatiert Steven Adams eine charakteristische Spannung, die beide Bücher durchziehe. Gemeint ist die Polarisierung zwischen einer Auffassung des Ornamentalen als einer "constant in the history of civilization ${ }^{1}$ und einer Betonung der Vielfalt in Form, Funktion und kultureller Bedeutung, die ornamentale Strukturen in unterschiedlichen historischen, soziokulturellen und ästhetischen Zusammenhängen auszeichnet. Was hier zur Sprache kommt, ist eine Spannung zwischen universalistischen und lokalistischen Leseweisen von Ornamenten: Bilden Ornamente so etwas wie eine anthropologische Konstante, "something supra-cultural [...], something visceral that speaks primarily to the body ${ }^{2}{ }^{2}$ wie der Rezensent Brett und Trilling paraphrasiert, oder sind sie lediglich als Epiphänomene spezifischer gesellschaftlicher Formationen anzusehen?

Angesichts dieser zugespitzten Fragestellung liegt eine versöhnliche - dialektische und teleologische - Antwort nahe. Diese könnte lauten: Man muss sich nicht entscheiden. Gerade in der geographischen Verschiedenheit und in der historischen Wandelbarkeit der Ornamente zeigen sich überzeitliche strukturelle Charakteristika und Funktionen des Ornamentalen. Anlässlich von Oleg Grabars verdienstvollem Buch The mediation of ornament (1992) gerät Rudolf Arnheim ins Schwärmen: „One receives a beau- tifully illustrated introduction to Islamic art, and each work earns its presence by serving to bring a theoretical issue to life. This is cross-fertilization at its very best «. ${ }^{3}$ Aus der kundigen Analyse islamischer Ornamente entspringt eine allgemeine Theorie visueller Sinnerzeugung durch abstrakte Formen überhaupt, und die pulsierenden Linien von Piet Mondrians Broadway Boogy Woogie treten neben den geschmückten Namen Alis, Schwiegersohn des Propheten und erster Imam, der das Cover von Grabars Buch ziert in Form

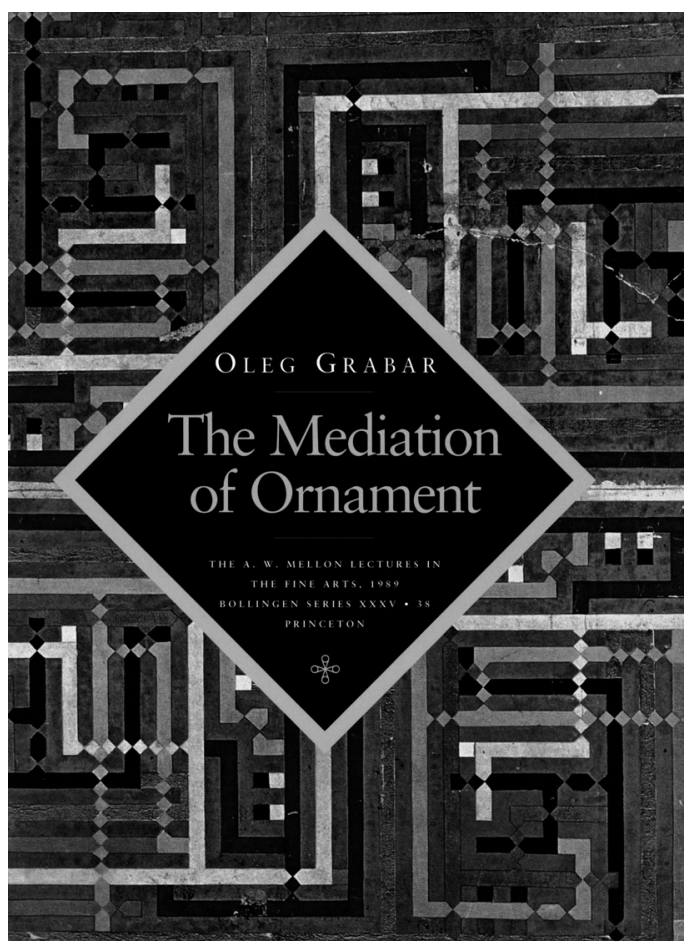

1 Oleg Grabar, The mediation of ornament, Princeton 1992, Cover: Buchmalerei auf Papier, 15. Jahrhundert, Iran. Istanbul, Topkapi-Serail Museum, Hazine 2152, fol. 9v 
eines "striking fifteenth-century Iranian painting whose combination of squares of seven different colors separated by black lines inevitably recalls some of Mondrian's best-known and most exciting compositions « (Abb. 1). ${ }^{4}$

Im Rahmen von Arnheims bzw. Grabars "cross-fertilization" oder wechselseitiger Befruchtung des Universalen und des Lokalen wächst dem Ornament eine privilegierte Rolle zu. Es scheint imstande, zwischen beiden Polen zu vermitteln, mehr noch, sie zu umgreifen und allgemeine anthropologische Strukturen, die mit den Eigentümlichkeiten unserer Wahrnehmung und unseres In-der-Welt-Seins als soziale Wesen zu tun haben, in spezifischer Gestalt zu verkörpern.

\section{II.}

Ein analoges Problem steht auch im Mittelpunkt der Kritik der Urteilskraft (1790) von Immanuel Kant, das Problem einer Kompatibilität zwischen konkreten sinnlichen Formationen, wie sie in der empirischen Welt aufgefunden werden - sei es im Bereich der Kunst oder im Bereich der Natur - mit allgemeinen Strukturen des ästhetischen Urteils. Obwohl sich `Ornamente dem Wort nach kein einziges Mal in der Kritik der Urteilskraft finden, handelt dieses Buch gleich auf mehrfache Weise von ornamentalen Strukturen.

A. Bekanntermaßen hat der Begriff des »Parergon" - als »Beiwerk" oder "Zierat" - aus dem berühmten $\S 14$, der überschrieben ist "Erläuterung durch Beispiele», seit Jacques Derridas Abhandlung Die Wahrheit in der Malerei (1978) einige Aufmerksamkeit erfahren. ${ }^{5}$

B. Doch nicht nur auf systematisch-begrifflicher Ebene, sondern auch auf der Ebene von Kants Beispielen tauchen Ornamente in vielerlei Gestalt auf. Als Paradigmen der "pulchritudo vaga«, der freien Schönheit, begegnen sie sowohl im Bereich des Naturschönen als auch des Kunstschönen, ${ }^{6}$ als »Zeichnungen à la grecque, das Laubwerk zu Einfassungen oder auf Papiertapeten ${ }^{7}$, in Form von »Lustgärten, Stubenverzierungen, allerlei geschmackvollem Geräte ${ }^{8}{ }^{8}$ als »der englische Geschmack in Gärten, der Barockgeschmack an Möbeln«.9 Sie klingen an in Kants Abneigung gegen "[a]lles Steif-Regelmäßige $\aleph^{10}$ oder gegen primordiale einfache geometrische Figuren wie Zirkel, Quadrat und Würfel. ${ }^{11}$ Im Namen der "pulchritudo vaga« lehnt Kant auch das Allee-Mäßige des umrankten Stangenwaldes der Pfeffergärten Sumatras ab, dessen allzu großer Regelmäßigkeit er »die dort an Mannigfaltigkeiten bis zur Üppigkeit verschwenderische Natur ${ }^{12}{ }^{2}$ vorzieht (Abb. 2).

Das erstaunlichste Beispiel aber schwebt auf eigentümliche Weise zwischen dem Kunstschönen und dem Naturschönen. Es betrifft eine kulturelle Praktik der Maori auf Neuseeland (Abb. 3). Man würde, so Kant, »eine Gestalt mit allerlei Schnörkeln und leichten, doch regelmäßigen Zügen, wie die Neuseeländer mit ihrem Tätowieren tun, verschönern können, wenn es nur nicht ein Mensch wäre «. ${ }^{13}$ Auf dieses Beispiel wird zurückzukommen sein.

C. Schließlich erfüllen die Beispiele der Kritik der Urteilskraft selbst - sie mögen von Ornamenten oder anderem handeln - im Rahmen der Gesamtarchitektur des Buches die Funktion eines Beiwerks im derridaschen Sinne: eines beunruhigenden, schwer festzumachenden Randphänomens, das nicht zur eigentlichen philosophischen Argumentation gehört, weil es aus empirischen und nicht aus transzendentalen Quellen stammt, das aber dennoch unverzichtbar für diese ist. Obwohl die Beispiele der Kritik der Urteilskraft zum größten Teil - von ein paar Ausflügen in die Antike abgesehen - der Lebenswelt des späten 18. Jahrhunderts entnommen sind, wobei wiederum der hohe Anteil an kolonialistischen Narrativen hervorsticht, ${ }^{14}$ sind sie als philosophisches Beiwerk dennoch unmittelbar mit der transzendentalen (also überzeitlichen, allgemeingültigen, apriorischen) Deduktion des Geschmacksurteils verwoben, 


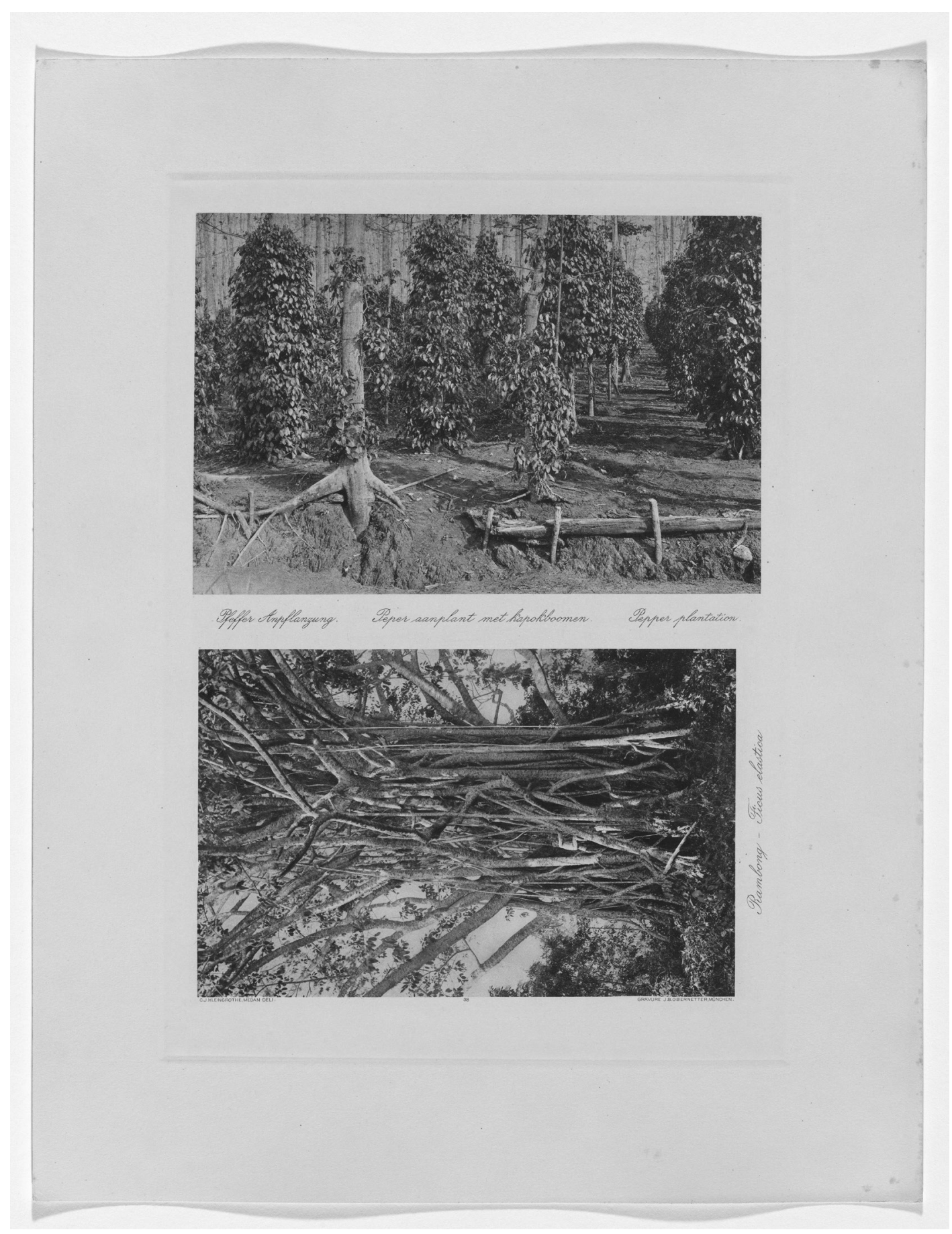

2 Charles Kleingrothe, Pfefferanpflanzung auf Indonesien, ca. 1903 -1920, Heliogravüre, 34,8 x 44,8 cm. Canberra, National Gallery of Australia 
wie sie die dritte Kritik versucht. Denn das Geschmacksurteil, zugleich »empirisch und ein einzelnes Urteil «, ${ }^{15}$ ist seiner Struktur nach stets auf konkrete Gegenstände bezogen.

Besonders im ersten Teil des Buches, der "Analytik des Schönen«, gibt Kant sich alle Mühe, seiner Argumentation dasselbe strenge Gepräge wie in der früheren Kritik der reinen Vernunft (1781) zu verleihen. So analysiert er das Geschmacksurteil nach den dort in der Kategorientafel verzeichneten allgemeinen Verstandesbegriffen der Qualität, der Quantität, der Relation und der Modalität. Diese Einteilung ist jedoch keineswegs zwingend für die kantische Deduktion des Geschmacksurteils, wie Derrida neben einer ganzen Reihe von anderen Autoren bemerkt hat. ${ }^{16}$ Derrida zufolge bildet die Kritik der reinen Vernunft vielmehr einen brüchigen, parergonalen Rahmen der Kritik der Urteilskraft. Die Ableitung der Eigenheiten des Geschmacksurteils aus der Kategorientafel reicht bei weitem nicht an die methodische Stringenz der Kritik der reinen Vernunft selbst heran und orientiert sich eher lose an deren Vorgaben, um spätestens nach der »Analytik des Erhabenen« aus dem Text zu verschwinden. Umso stärker sticht die Präsenz der kantischen Beispiele ins Auge. Wenn man den Text aufmerksam liest, liegt die Vermutung nahe, dass sie das, was nicht mit bestimmten Begriffen gefasst werden kann, anschaulich machen und exemplarisch vor Augen stellen sollen.

\section{III.}

Auf welche Weise wird aber das Geschmacksurteil in der »Analytik des Schönen « behandelt? Welche Beziehung hat es zum Ornamentalen, das unter dem Namen »Zierat" und »Parergon" sowie in Form von Kants eigenen Beispielen aufgerufen wird? Und ist die Beispielfülle der Kritik der Urteilskraft eine primär didaktische Maßnahme des Autors, um ausgesprochen abstrakte, transzendentale Gedankengänge zu illustrieren und besser verständlich zu machen, oder hat diese Fülle mit der Struktur des Geschmacksurteils selbst zu tun?

Beginnen wir zunächst, ausgehend von der »Analytik des Schönen" und der ästhetischen Urteilskraft, die darin verhandelt wird, mit einer kurzen Darlegung der Problemstellung Kants: Das Geschmacksurteil schreibt einem beliebigen Gegenstand das Prädikat der Schönheit zu. Seine paradigmatische Form lautet: »Dieser Gegenstand ist schön«. Anders als bei einem gewöhnlichen empirischen Urteil - etwa wenn ich sage: »Dieses Tier ist eine Katze« - gibt es Kant zufolge jedoch keinen vorgängigen apriorischen und auch keinen empirischen Begriff, der bestimmen würde, wie genau ein schöner Gegenstand auszusehen hat und nach welchen Regeln er als schön zu beurteilen ist. Von der Katze kann man das sagen: "Sie ist ein haariges Tier auf vier Beinen mit Schnurrhaaren und Krallen, ihre Augen funkeln in der Nacht und im Gegensatz zum Hund miaut sie.« Im Urteil über eine Katze als Katze ist deshalb die »bestimmende Urteilskraft« tätig, wie Kant sagt, sie bestimmt dieses Tier nach bestimmten allgemeinen Merkmalen als Katze. »Ist das Allgemeine (die Regel, das Prinzip, das Gesetz) gegeben, so ist die Urteilskraft, welche das Besondere darunter subsumiert, [...] bestimmend. Ist aber nur das Besondere gegeben, wozu sie das Allgemeine finden soll, so ist die Urteilskraft bloß reflektierend ${ }{ }^{17}{ }^{17}$

Im Geschmacksurteil tritt die Urteilskraft als »reflektierende» in Erscheinung. Sie geht von einem konkreten, bestimmten Gegenstand aus und beurteilt diesen als schön. Sie vollzieht den Sprung von einer individuellen sinnlichen Erscheinung in der Anschauung zum allgemeinen Prädikat der Schönheit.

Wie geht sie dabei vor? Wie bereits angedeutet, konstituiert das Prädikat der Schönheit keinen Begriff, den man a priori oder a posteriori definieren könnte, um dann diejenigen Gegenstände auszuwählen, die unter diesen Begriff fallen. Wie kann ich dann sagen, ein bestimmter 
Gegenstand sei schön? Kant gibt eine ebenso verblüffende wie originelle Antwort: Ich beurteile eigentlich nicht die Eigenschaften des Gegenstandes, sondern ein Zusammenstimmen meiner Erkenntnisvermögen Einbildungskraft und Verstand, in das mich die Betrachtung dieses Gegenstandes versetzt. Dieses Zusammenstimmen wird durch ein Gefühl der Lust ${ }^{18}$ angezeigt. Es ist wesentlich, dass "Lust» hier kein mit der Befriedigung eines Bedürfnisses oder der Steigerung eines Reizes verbundenes Wohlgefühl meint. Empfinde ich beim Anblick eines Vogels oder einer Statue des Marsyas dieses Gefühl, dann urteile ich mit Recht, der Vogel oder die Statue seien schön. Schönheit im Sinne Kants ist also in erster Linie eine Eigenschaft des Subjektes und wird erst sekundär demjenigen konkreten Objekt, das in mir das Gefühl der Lust ausgelöst hat, zugeschrieben. Was das Geschmacksurteil in Reinform betrifft, unterscheidet Kant konsequenterweise auch nicht zwischen Schönheiten der Natur und Schönheiten der Kunst. Da sich von vornherein keine notwendigen Merkmale eines schönen Gegenstandes angeben lassen und auf die Reaktion des eigenen Gefühls gewartet werden muss, bevor man ihn als schön beurteilen kann, lassen sich auch keine Merkmale der spezifischen Schönheit von Kunst- oder Naturprodukten angeben.

Worauf aber verweist das anlässlich eines schönen Gegenstandes unmittelbar empfundene Gefühl der Lust? Es verweist auf einen besonderen Zustand - eine "Proportion $«^{19}$ - unserer Erkenntniskräfte, denjenigen nämlich, mittels dessen wir erkennen, dass ein Gegenstand unserer Wahrnehmung, noch bevor wir ihn mit dem Verstand explizit begrifflich bestimmen, eine sinnliche Ordnung oder Konfiguration aufweist - Kant sagt: eine "Zusammenstimmung des Mannigfaltigen $\ll^{20}$-, die uns zugänglich ist. Mit anderen Worten: Wir erfahren, dass dies ein Ding - »unbestimmt, was es sein solle " $^{21}$ ist, das wir überhaupt mit der Einbildungskraft auffassen und mittels des Verstandes beurteilen können, indem wir Begriffe in Anschlag bringen. Das Gefühl der Lust bezieht sich also auf die Angemessenheit der Welt zu unseren Erkenntnisvermögen. Es ist das triumphierende Gefühl dessen/derjenigen, der/die tatsächlich innewird, wie sich eine heterogene Mannigfaltigkeit, die nicht er/sie selbst ist, die ihm/ihr zustößt und über die er/sie keine Macht hat, unwillkürlich ordnet und Erkennbarkeit verspricht. In der Kritik der reinen Vernunft hatte Kant die prinzipielle, bloß apriorische Ordnungsleistung unserer Erkenntniskräfte beschrieben. Im Gefühl der Lust wird diese Ordnungsleistung angesichts des materiellen Chaos der Empfindungen und der "Zufälligkeiten ${ }^{22}$ der empirischen Welt unmittelbar für jeden und jede erfahrbar.

IV.

Wo ist nun der Platz der Ornamente, Parerga, Zierrate, Laubwerke, Lustgärten, Pfeffergärten, Stubenverzierungen, Schnörkel auf Möbeln oder in Gesichtern in dieser Konstellation? Sie antworten auf ein fundamentales Paradoxon in der Beurteilung des Schönen: Schönheit ist keine Eigenschaft von Gegenständen, sondern bezeichnet ein spezifisches Lustgefühl im wahrnehmenden Subjekt. Dieses entsteht jedoch nicht völlig unabhängig von Gegenständen. Es wird vielmehr durch diese ausgelöst und ihnen im Geschmacksurteil reattribuiert. Obwohl ich sagen müsste: »Ich empfinde ein Gefühl der Lust bei der Wahrnehmung dieses Gegenstandes", sage ich: »Dieser Gegenstand ist schön«. Aber lösen nicht manche Gegenstände eher als andere das Gefühl ästhetischer Lust in uns aus; gibt es nicht bestimmte Traditionen und Gebrauchsweisen des Schönen, die an ganz bestimmte, benennbare Gegenstände oder Gegenstandsklassen gebunden sind? Einerseits ist Schönheit nicht von vornherein begrifflich bestimmbar und kann immer erst nachträglich - Kant sagt: »reflektierend" - von einem Gegenstand, der 
eine bestimmte Lust erzeugt hat, ausgesagt werden. Andererseits gibt es $\mathrm{zu}$ bestimmten Zeiten und an bestimmten Orten faktische Konventionen und Übereinkünfte darüber, welche Objekte als schön gelten und welche nicht, oder zumindest, welche Objekte eher als schön gelten können als andere, und Kant ist sich dieser Tatsache bewusst.

Was also benötigt wird, sind Mittelglieder zwischen der subjektiv gefühlten Lust des Schönen und den Objekten, die irgendwie mit dieser Lust verbunden sind. Ein privilegiertes dieser Mittelglieder, so die These, die hier verfolgt werden soll, stellen ornamentale Strukturen dar. Ornamentale Formen eröffnen in der Kritik der Urteilskraft die Möglichkeit, im Rahmen einer Theorie des Schönen nichtobjekthafte empirische Erscheinungen, mithin etwas wie eine rabstrakter Gegenständlichkeit zu denken. So stellt David Morgan Kant nicht zu Unrecht an den Beginn einer mehr oder weniger >deutschen $<\mathrm{Ge}$ schichte der Abstraktion, die ihre wesentlichen Antriebe aus der theoretischen und praktischen Autonomisierung von Ornamenten gewinnt und in das Denken von Wilhelm Worringer und Wassily Kandinsky mündet. Morgan stützt sich nicht zuletzt auf die kantische Unterscheidung von "pulchritudo vaga und "pulchritudo adhaerens « - freier und anhängender Schönheit - aus dem $₫ 16$ der Kritik der Urteilskraft. ${ }^{23}$

V.

Schon in $\$ 14$ war Kant bei der Erläuterung der dem Geschmacksurteil eigenen Zweckmäßigkeit ohne Zweck auf die Zierrate oder Parerga zu sprechen gekommen. Er erwähnt sie dort im Rahmen einer folgenschweren Privilegierung der reinen Form eines Gegenstandes gegenüber seinen Farben im ästhetischen Urteil. Nur die Form eines Dings vermag ein von allem empirischen Wohlgefallen freies ästhetisches Lustgefühl zu erzeugen; Farben (außer vielleicht reine ungemischte Farben) reizen indes bloß die Sinne und erwecken unser Interesse. Demnach ist es in der bildenden Kunst die "Zeichnung " $^{24}$ (und nicht die Farbe) und in der Musik die Komposition $^{25}$ (und nicht die Töne), die geeignet sind, eine Empfindung des Schönen hervorzurufen. Hier heißt es nun: „Selbst was man Zieraten [sic] (Parerga) nennt, d.i. dasjenige, was nicht in die ganze Vorstellung des Gegenstandes als Bestandstück innerlich, sondern nur äußerlich als Zutat gehört und das Wohlgefallen des Geschmacks vergrößert, tut dieses doch auch nur durch seine Form, wie Einfassungen an Gemälden, Gewänder an Statuen oder Säulengänge an Prachtgebäuden «. ${ }^{26}$

Auch die Zierrate also, die nicht eigentlich zu einem Gegenstand seiner Substanz und seinem Begriff nach gehören, die ihn lediglich umspielen, sind fähig, ein Geschmacksurteil hervorzurufen. Allerdings gilt für sie dieselbe Einschränkung wie für Gegenstände im eigentlichen Sinn - sie vermögen ausschließlich kraft ihrer Form, schön zu sein: Ein goldener Rahmen erhöht nur den sinnlichen Reiz eines Gemäldes, er ist bloßer "Schmuck ${ }^{27}$ und trägt nichts zum Gefühl von dessen Schönheit bei.

In $\$ 14$ erwähnt Kant ausschließlich RahmenRand- oder Umhüllungsphänomene als Beispiele für Zierrate/Parerga: Gemälderahmen, Gewänder von Statuen und - Vitruv folgend Säulengänge, die Gebäude wie ein Kleid umhüllen. In seiner differenztheoretischen Auslegung des Parergons fasst Derrida dieses denn auch beinahe durchgehend als Rahmenstruktur, eine Interpretation, die ihren historischen Ausgangspunkt in der Schrift Vorbegriffe zu einer Theorie der Ornamente von Karl Philipp Moritz hat, der 1793 - wenige Jahre nach dem Erscheinen der ersten Auflage der Kritik der Urteilskraft - das Ornament als ein Prinzip der Isolation deutete und es unmittelbar an die Funktion des Bilderrahmens band. ${ }^{28}$

Sieht man sich die zeitgenössischen Semantiken von "Zierat« bzw. »Parergon« an, so stellt 
man allerdings fest, dass nicht nur Rahmenstrukturen gemeint waren. Ein Blick in das Goethe-Wörterbuch zeigt zudem, dass »Parergon/Beiwerk" und "Zierat" keineswegs Synonyme darstellten, wie Kant dies anzunehmen scheint, wenn er in der zweiten Auflage der Kritik der Urteilskraft von 1793 den Ausdruck »Parerga" in Klammern und ohne weiteren Kommentar zu »Zieraten « hinzufügt. ${ }^{29}$ »Beiwerk" oder »Parergon« bedeutete nämlich auch die durchaus figürlichen Nebengegenstände in einem Bild, die vor dem Hauptgegenstand zurücktraten und häufig kleiner als dieser in der Bildtiefe dargestellt waren. Unter dem Lemma »Zierat" hingegen listet das Grimmsche Wörterbuch eine Fülle von Bedeutungen auf. Es werden erwähnt: Schmuck, Schmuckstück, Verzierungen oder einzelne Zierelemente an Bauwerken, die Ausstattung von Gebäuden wie Wandgemälde, gemalte Ornamente oder Zierlinien, natürlicher oder künstlicher Schmuck des menschlichen Körpers, von Tieren oder von Pflanzen, Auszierungen der Schrift oder des musikalischen Vortrags, manierierte, vielfach überladene Kunstwerke. ${ }^{30}$

\section{VI.}

Wenden wir uns jetzt dem von Morgan zitierten $\$ 16 \mathrm{zu}$, in dem Kant zwei Arten von Schönheit unterscheidet - die »pulchritudo adhaerens« oder "anhängende« Schönheit und die "pulchritudo vaga«, also »umherschweifende«, "flüchtige«, "ungebundene«, kurz "freie« Schönheit. Erstere setzt einen Begriff davon voraus, was ein Gegenstand sein soll, begründet also keine Schönheit im Sinne des reinen Geschmacksurteils. Sie ist an die "Vollkommenheit" oder »objektive innere Zweckmäßigkeit « ${ }^{31}$ eines Gegenstandes gebunden. Beispielsweise werden einfache geometrische Figuren wie Kreis, Quadrat und Würfel genau dann als schön angesehen, wenn sie perfekt um einen Mittelpunkt gerundet sind bzw. exakt gleichlange, im Winkel von neunzig
Grad aufeinander stehende Kanten aufweisen. ${ }^{32}$ "Niemand wird leichtlich einen Menschen von Geschmack dazu nötig finden, um an einer Zirkelgestalt mehr Wohlgefallen als an einem kritzlichen Umrisse, an einem gleichseitigen und gleicheckigen Viereck mehr als einem schiefen, ungleichseitigen, gleichsam verkrüppelten $\mathrm{zu}$ finden $\ll^{33}$

Freie Schönheiten hingegen sind »für sich bestehende ${ }^{34}$ Schönheiten, die nicht aufgrund ihrer Zweckmäßigkeit bzw. eines vorgängigen Begriffs, an dem sie gemessen werden und dem sie entsprechen sollen, als schön beurteilt werden. Kant spricht von einer «Zusammensetzung des Mannigfaltigen «, ${ }^{35}$ die aus der bloßen Konstellation ihrer Formen eine sinnliche, nichtbegriffliche Kohärenz erzeugt. Und wie fast immer in der Kritikder Urteilskraft lässt er Beispiele folgen. Hier finden sich in langer Aufzählung fast alle der im Grimmschen Wörterbuch versammelten zeitgenössischen Bedeutungen von "Zierat«: »Blumen sind freie Naturschönheiten $«{ }^{36}$ Aber auch: »Viele Vögel (der Papagei, der Kolibri, der Paradiesvogel), eine Menge Schaltiere des Meeres sind für sich bestehende Schönheiten, die gar keinem nach Begriffen in Ansehung seines Zwecks bestimmten Gegenstande zukommen, sondern frei und für sich gefallen. So bedeuten die Zeichnungen à la grecque, das Laubwerk zu Einfassungen oder auf Papiertapete usw. für sich nichts; sie stellen nichts vor, kein Objekt unter einem bestimmten Begriffe, und sind freie Schönheiten. Man kann auch das, was man in der Musik Phantasien (ohne Thema) nennt, ja die ganze Musik ohne Text zu derselben Art zählen «. ${ }^{37}$

Während in $\$ 14$ ornamentale Strukturen nur am Rande vorkommen, als unselbstständige, äußerliche, akzidentelle, verzichtbare "Zutaten" von Gegenständen, um zu zeigen, dass selbst sie - die Nebensachen, Zierrate und Beiwerke - als schön empfunden werden können, sofern man sie nach ihrer Form (und nicht etwa der goldenen Farbe nach) beurteilt, rücken sie in $\S 16$ - und 
noch einmal in der "Allgemeinen Anmerkung zum ersten Abschnitte der Analytik « ${ }^{38}$ - in das Zentrum der Argumentation und steigen zu Paradigmen der freien Schönheit auf. Dabei kommt es zu einer bemerkenswerten Umwertung: Das Anhängende, Nebensächliche, nicht eigentlich zum Gegenstand Gehörige - und diese wörtliche ist die relevante Bedeutung von "Parergon" in der Kritik der Urteilskraft - steigt auf zum Inbegriff sinnlich wahrnehmbarer Schönheit, die dem reinen Geschmacksurteil angemessen ist. Die eigentliche Substanz hingegen bzw. der Begriff davon, was einen Gegenstand ausmacht, insofern er ein Gegenstand einer bestimmten Art ist, vermag bloß eine nachgeordnete, eben dem Begriff anhängende Schönheit zu begründen.

Als Kant die Kritik der Urteilskraft verfasste, war das von Juste-Aurèle Meissonier in den 1730er-Jahren eingeführte Rocaille-Ornament, jenes asymmetrische Muschelornament, das namensgebend für die Epoche des Rokoko werden sollte, im deutschsprachigen Raum bereits Objekt einer jahrzehntelangen ästhetischen Debatte gewesen. ${ }^{39}$ Einerseits war der Rocaille-Stil um die Jahrhundertmitte in Architektur und Dekorationskunst prägend gewesen, wie nicht zuletzt die Fülle von Ornamentstichen belegt, andererseits war die Rocaille seit den 1750er-Jahren das Ziel kunsttheoretischer Angriffe aus dem Bereich des deutschen Frühklassizismus gewesen. Autoren wie Friedrich August Krubsacius oder Johann Joachim Winckelmann hatten sowohl die wuchernde, jedes rationale Maß übersteigende Regellosigkeit der Rocaille als auch ihre zu Hybridbildungen aller Arten und Gattungen neigende Unnatürlichkeit einer ausführlichen Kritik unterzogen.

Die zitierten Passagen aus $\$ 16$ spielen freilich auf das ins Natürliche gewendete Motiv der Rocaille - »eine Menge Schaltiere des Meeres" - ebenso an wie auf die gegen Ende des 18. Jahrhunderts in Deutschland zunehmend beliebten Wanddekorationen im Stil von Herculaneum und Pompeji - "Zeichnungen à la grecque».
Im späteren Kapitel »Allgemeine Anmerkung zum ersten Abschnitte der Analytik« überwiegt dann eindeutig das Rokokohafte: "Lustgärten", »Stubenverzierung«, Vermeidung der »Regelmäßigkeit, die sich als Zwang ankündigt« und »der Barockgeschmack an Möbeln «. ${ }^{40}$ Kants Katalog der Beispiele stellt somit zwei Formen des Ornaments nebeneinander, die eine wichtige historische Differenz aufweisen: Während die Rocaille eng mit einer höfisch-absolutistischen Gesellschaftsordnung verbunden ist, kündigt sich in den aufgeräumten Bildfeldern im pompejanischen Stil, den selbst Winckelmann zu schätzen wusste, ${ }^{41}$ ein bürgerlicher Geschmack an. Wie ist diese Inkonsistenz von Beispielen historischer Ornamenttypen zu werten? Es ist klar, dass Kant die Rokoko-Ornamente nicht in ihrer konkreten historischen Dimension nahm, auch wenn er sich dieser bewusst gewesen sein mag. Vielmehr ist zu vermuten, dass das historische Verblassen des Stilparadigmas der Rocaille dieses für Kant in systematischer Hinsicht verfügbar machte, um - entgegen der klassizistischen Furcht vor Regel- und Maßlosigkeit - die besondere Freiheit der Einbildungskraft im Spiel der Erkenntnisvermögen zu exemplifizieren.

Die Bestimmung der Zierrate im Allgemeinen als Beispiele der freien Schönheit fasst Helmut Pfotenhauer wie folgt zusammen: "Kant rechnet sie [...] nicht mehr, wie bisher üblich, zu den anhängenden Schönheiten, sondern zu den frei vagierenden; er kehrt also ihre Bedeutung genau um und betont nicht mehr ihre Unterordnung, sondern ihre Entlastung vom Gegenständlich-Repräsentativen und Funktionalen $« .^{42}$ Es scheint, als böten die Zierrate in der Kritik der Urteilskraft die Möglichkeit, die apriorischen kategorialen Merkmale des Geschmacksurteils in der empirischen Welt wiederzufinden und so die Kluft zumindest reflexiv zu überbrücken, welche die Dissoziierung von Schönheit und Objekt und die alleinige Zuschreibung des Prädikats des Schönen an ein Lustgefühl des Subjekts gerissen hatten. 
Man könnte versucht sein, die spezifische Weise, wie die Kategorien der Quantität, Qualität, Relation und Modalität bei Kant das Geschmacksurteil strukturieren, in den Formen der Zierrate wiederzufinden. Wie auf subjektive Weise im Geschmacksurteil ist auch in den Zierraten als Elementen der empirischen Welt, die gleichwohl keine Gegenstände darstellen, eine Zweckmäßigkeit ohne bestimmten Zweck ausgedrückt (Kategorie der Relation). Ihre Existenz ist belanglos. $\mathrm{Ob}$ es sie gibt oder nicht, macht keinen Unterschied (Kategorie der Qualität). Da sie keine bestimmten Gegenstände vorstellen, handelt es sich um allgemeine Strukturen (Kategorie der Quantität), die zu verschiedenen Zeiten und in verschiedenen Kulturen mit anthropologischer Notwendigkeit auftauchen und die in der Natur das formale Gerüst des tierischen und pflanzlichen Lebens zu bilden scheinen (Kategorie der Modalität).

\section{VII.}

Nachdem die systematische Stellung der "Zieraten (Parerga) « ${ }^{43}$ in der Kritik der Urteilskraft erörtert wurde, gilt es, die Beispiele Kants in den Blick zu nehmen, welche die apriorischen Ableitungen der Charakteristika des Geschmacksurteils wie ein Beiwerk umgeben. Handelt es sich um ein notwendiges oder zufälliges Beiwerk? Sind die Beispiele bloße Illustration, also "Schmuck ${ }^{44}$ (in Kants eigener Terminologie), oder sind sie in gewisser Weise unentbehrlich?

Das Geschmacksurteil ist nach Kant exemplarisch. Da das Geschmacksurteil nicht nach einem vorgängigen Begriff oder irgendwelchen Schönheitsregeln urteilt oder auch nur urteilen kann, ein Gegenstand sei schön, sondern allein nach dem von der Wahrnehmung dieses Gegenstandes bewirkten Gefühl der Lust, lässt sich die Schönheit des Gegenstandes immer erst nachträglich feststellen, wenn der Gegenstand die Lust schon bewirkt hat. Dann aber ist dieser
Gegenstand ein »Beispiel« der Schönheit. Das Geschmacksurteil ist ein »einzelnes Urteil « ${ }^{45}$ es bezieht sich auf einen bestimmten Gegenstand, so wie dieser gerade ist. Die Urteilskraft ist im Geschmacksurteil reflektierend, das heißt, sie sucht eine "mögliche" Regel zu einem gegebenen Einzelfall. So schreibt Kant 1789 in der sogenannten »Ersten Einleitung « in die Kritik der Urteilskraft: "Reflectiren (Überlegen) aber ist: gegebene Vorstellungen entweder mit andern, oder mit seinem Erkenntnißvermögen, in Beziehung auf einen dadurch möglichen Begriff, zu vergleichen und zusammen zu halten ${ }^{46}{ }^{46}$

Der im Geschmacksurteil als schön beurteilte Gegenstand wird »wie ein Beispiel einer allgemeinen Regel, die man nicht angeben kann, angesehen «. ${ }^{47}$ Könnte man die Regel ein für alle Mal angeben, hätte man ein allgemeingültiges apriorisches Rezept, wie schöne Gegenstände $\mathrm{zu}$ beurteilen und herzustellen sind. Gerade das aber würde jede Art der höchsten, der freien Schönheit verunmöglichen.

\section{VIII.}

Wie kann sichergestellt werden, dass die einzelnen »Beispiele« der Schönheit in den einzelnen Geschmacksurteilen, sofern sie nicht bloß von ornamentalen Strukturen inspiriert sind, konvergieren? Wie lässt sich erklären, dass es eine gewisse Konstanz in den Zuschreibungen der Schönheit an bestimmte Gegenstände gibt - zumindest zu gewissen Zeiten und an bestimmten Orten? Wie lässt sich erklären, dass verschiedenen Menschen ein und dieselbe Sache schön finden oder derselbe Mensch zu verschiedenen Zeiten? Handelt es sich hierbei um groteske $\mathrm{Zu}$ fälle?

Um solche Aporien zu vermeiden, postuliert Kant, das Geschmacksurteil habe einen allgemeinen Anspruch, es fordere "die Beistimmung aller «, ${ }^{48}$ obwohl es auf einem subjektiven Gefühl der Lust gründet. Wenn ich sage »dieses ist ein 
schöner Garten«, so meine ich nicht, der Garten sei nur für mich schön. Der allgemeine, apriorische Anspruch, der in solchen Geschmacksurteilen zum Tragen kommt, wird zum einen formal begründet: Das Gefühl der Lust verweist auf ein harmonisches Verhältnis der subjektiven Erkenntnisvermögen Einbildungskraft und Verstand, das grundsätzlich allen Urteilen bei allen Menschen, wenn auch athematisch, zugrunde liegt: „Diese Lust muß notwendig bei jedermann auf den nämlichen Bedingungen beruhen, weil sie subjektive Bedingungen der Möglichkeit einer Erkenntnis überhaupt sind, und die Proportion dieser Erkenntnisvermögen, welche zum Geschmack erfordert wird, auch zum gemeinen und gesunden Verstande erforderlich ist, den man bei jedermann voraussetzen darf $«{ }^{49}$

Diese bloß formale Allgemeinheit muss jedoch zum anderen durch allgemeingültige Beispiele der Schönheit konkretisiert werden. Hier dringt ein schwacher empirischer Reflex in den Formalismus des Geschmacksurteils ein: »[...] die Einhelligkeit, soviel als möglich, aller Zeiten und Völker in Ansehung dieses Gefühls in der Vorstellung gewisser Gegenstände ist das empirische, wiewohl schwache und kaum zur Vermutung zureichende Kriterium der Abstammung eines so durch Beispiele bewährten Geschmacks von dem tief verborgenen, allen Menschen gemeinschaftlichen Grunde der Einhelligkeit in Beurteilung der Formen, unter denen ihnen Gegenstände gegeben sind.$^{50}$

Der durch Beispiele bewährte Geschmack legt für alle Zeiten und für alle Völker "Muster « ${ }^{51}$ fest für das, was als schön gelten kann. Für die Dichtkunst sieht Kant in den "toten und gelehrten Sprachen ${ }^{52}$ ein solches Muster: Ihr Lexikon ist stabil und ihre Grammatik verändert sich nicht. Der Geschmack tritt hier in doppelter Funktion auf - einmal in transzendentaler Bedeutung als "Gemeinsinn", mithin als eine Vernunftidee, welche die Allgemeinheit des Geschmacksurteils jenseits aller "subjektiven Privatbedingungen « begründet..$^{53}$ In dieser Hinsicht ist der Geschmack - analog zum kategorischen Imperativ - rein formal bestimmt, er «abstrahiert« von allen kontingenten, bloß subjektiven Bedingungen, von $»$ Reiz und Rührung . $^{54}$ Andererseits wird der Geschmack in historischempirischer Perspektive gefasst und auf "Zeiten und Völker« bezogen. Die Verbindung zwischen dem transzendentalen und dem empirischen Bereich leisten die Beispiele oder Muster des guten Geschmacks, die in dieser Hinsicht eine analoge Funktion zu den Zierraten in $\$ 16$ einnehmen. Das Problem dieser, im Tonfall vorsichtig vorgetragenen Argumentation, liegt freilich darin, dass die rein formale Normativität des Geschmacksurteils mit einer bestimmten historischen Faktizität verbunden wird, der eines klassizistischen, europäischen Geschmacks. Dieser Zusammenhang wird uns im Folgenden beschäftigen.

Kant zufolge gibt es ein höchstes Muster oder "Ideal der Schönheit« von universaler Gültigkeit. ${ }^{55}$ Ein solches Urbild des Geschmacks kann nicht empirisch, sondern nur als Idee existieren. Da sich das Geschmacksurteil auf sinnliche Anschauungen bezieht, kann dieses Urbild wiederum nur als "Vorstellung eines einzelnen als einer Idee adäquaten Wesens" in der Einbildungskraft bestehen, mit anderen Worten als »Ideal.$^{56}$ Dieses Ideal aber ist der Mensch. Seine Gestalt fungiert als der »sichtbare Ausdruck sittlicher Ideen «. ${ }^{57}$ Nur der Mensch vermag sich nach Kant seine Zwecke selbst zu setzen, eine sittliche Freiheit, die der ästhetischen des Geschmacksurteils analog ist und sich in dieser symbolisiert. Das Schöne ist nach Kant in letzter Instanz ein Reflex der unverbrüchlichen Sittlichkeit des Menschen, das heißt der Freiheit, sein Begehrungsvermögen durch das Vernunftgesetz des kategorischen Imperativs und nicht durch Begierden und sinnliche Triebfedern bestimmen zu lassen. Der Mensch ist auf diese Weise sowohl Subjekt - als Einzelwesen - als auch privilegiertes Objekt des Geschmacksurteils - als Ideal der Schönheit. 
Hier nun wird der Unterschied zwischen freier und anhängender Schönheit relevant. Anders als die Zierrate als Paradigmen der freien Schönheit fällt das Ideal der Schönheit in den Bereich der "pulchritudo adhaerens", denn die Schönheit eines Menschen "setzt einen Begriff vom Zwecke voraus, was das Ding sein soll, mithin einen Begriff seiner Vollkommenheit «.58 Der systematischen Differenz zwischen freier und anhängender Schönheit bei Kant entspricht auf der Ebene der Beispiele die historisch-stilistische Differenz zwischen Rokoko und Frühklassizismus, zwischen dem Spiel der Ornamente und der in sich ruhenden Würde der menschlichen Gestalt, eine Differenz, die bei Winckelmann und Lessing als Gegensatz zwischen dem Beiwerk der Bekleidung und der nackten Schönheit menschlicher Form verhandelt wurde. ${ }^{59}$

\section{IX.}

Wir haben gesehen, dass jegliches Geschmacksurteil - ob im Bereich der freien oder der anhängenden Schönheit - exemplarisch verfährt und im Prinzip einen beliebigen Gegenstand der empirischen Welt als schön zu beurteilen vermag, wobei die anhängende Schönheit einen Begriff seiner Zweckmäßigkeit voraussetzt. Wir haben weiters gesehen, dass Kant zufolge die faktische historische Synchronisierung der Beispiele und ihre Orientierung an Mustern des Geschmacks als ein »schwaches Kriterium« für die notwendige, transzendentale Allgemeinheit des Geschmacksurteils genommen werden kann, die ihrerseits auf die transzendentale Ausstattung aller Menschen mit denselben Erkenntnisvermögen verweist.

Obwohl es prinzipiell denkbar wäre, sich auf eine rein formale Bestimmung des Geschmacksurteils zu beschränken, ist die Kritik der Urteilskraft voller Beispiele des Schönen, als hätte es nicht genügt, das Geschmacksurteil rein der Form nach zu fassen, sondern als würde seine exemplarische, auf einzelne, empirische Gegenstände gehende Struktur auch eine konkrete, beispielhafte Nennung solcher Gegenstände erfordern. Es ist davon auszugehen, dass Kant seine eigenen Beispiele nicht als ein beliebiges Sammelsurium verstand, sondern ihnen im Sinne eines "schwachen Kriteriums" selbst paradigmatischen Charakter zuerkannte, um die transzendentale Struktur des Geschmacksurteils reflexiv in seiner anthropologischen Dimension zu spiegeln.

Gerade im Bereich des reinen ästhetischen Urteils und der mit ihm korrespondierenden freien Schönheiten haben Kants Beispiele selbst den Status von Mustern, von Paradigmen des Geschmacks, so wie er in der Geschichte der Menschheit zu Tage tritt. Die Abundanz von Ornamenten und abstrakten, mehrfach geschwungenen Linien sowie die vielen aus dem Bereich des Naturschönen und der fernen Kolonien genommenen Beispiele verweisen auf das Bemühen, auch auf der Ebene der Beispiele - der faktischen Exempel für Schönheit - eine für alle Völker und alle Zeiten verbindliche Allgemeinheit auszudrücken. Eine wilde Tulpe, eine Muschel, ein Paradiesvogel, ein Kolibri, ein Papagei sind für alle Menschen und zu allen Zeiten schön. Oder nicht? Haben sich nicht alle Kulturen auf allen Erdteilen an "Schnörkeln und leichten, doch regelmäßigen Zügen $\aleph^{60} \mathrm{er}-$ freut?

Kants Beispiele, zumal wenn sie Ornamente betreffen, sind in einer Indifferenzzone von Kultur und Natur angesiedelt, die sie der historischen Veränderung scheinbar entzieht und in ihrer Normativität befestigt. ${ }^{61}$ Die "toten und gelehrten Sprachen" sind zwar eindeutig Menschenwerk, doch bilden sie durch ihr Vergangen-Sein, durch die Abgeschlossenheit ihres geschichtlichen Werdens ein quasinatürliches Substrat für die lebendigen Sprachen. Die künstlichen Ornamente auf Tapeten oder an Möbelstücken sehen aus wie naturhafte Formen, andererseits wird die Natur in europäischen 
Lustgärten oder auf indonesischen Pfefferplantagen von Menschenhand in Form gezwungen. Mehr noch: Auch die Gegenstände der unberührten Natur, sofern sie schön sind, geben der Auffassung des Menschen "gerade eine solche Form an die Hand [...], die eine Zusammensetzung des Mannigfaltigen enthält, wie sie die Einbildungskraft, wenn sie sich selbst frei überlassen wäre, in Einstimmung mit der Verstandesgesetzmäßigkeit überhaupt entwerfen würde . $^{62}$ In der Folge spricht Kant von einer »Technik der Natur,$^{63}$ ja von einem »Begriff von eben derselben als Kunst ${ }^{6}{ }^{64}$ Diese Rede ist freilich im Sinne der reflektierenden Urteilskraft zu verstehen, im Modus des rals ob<, sie setzt aber, ebenso wie die Definition des Kunstwerks - »schöne Kunst muss als Natur anzusehen sein, ob man sich ihrer zwar als Kunst bewußt ist ${ }^{65}$ - die zumindest formale Vertauschbarkeit von »Kunst« und »Natur« voraus.

Wie Richard Heinrich dargelegt hat, lässt sich die Indifferenz von Kunst und Natur, die sich sowohl in den methodischen Überlegungen als auch in den Ornament-Beispielen der Kritik der Urteilskraft artikuliert, als die spezifische theoretische Signatur eines Zeitalters, des Rokoko, verstehen: "Die Differenz von Bild und Ornament ist unentscheidbar geworden. Dieses Spiel mit den Grenzen entfaltet sich aber im Laufe des Jahrhunderts in eine bestimmte Richtung, nämlich zur Natur hin. Man könnte das auf verschiedene Weisen deutlich machen, am eindrucksvollsten gewiss anhand des Schicksals des typischen und namengebenden OrnamentMotives selbst, der Muschelform der Rocaille. Die Muschel ist keineswegs eine ins ornamentale Repertoire übernommene Naturform, sondern wurde zuerst aus anderen Ornamentformen wie der Palmette entwickelt; erst allmählich erwarb sie sich ihr eigenes snatürliches Motiv hinzu. [...] Das Rokoko erscheint so als Dynamik, die die rationale Autonomie des Ornamentalen schrittweise transformiert: zuerst in die referentielle Struktur von Abbildung und dann, nach deren Dekonstruktion, in übergreifende Ganzheiten von Natur und Leben «. ${ }^{66}$

$\mathrm{X}$.

Die Beispiele aus der Kritik der Urteilskraft tragen das deutliche Gepräge der kantischen Lebenswelt des späten 18. Jahrhunderts. Während die Ornamente, auf die sich Heinrich bezieht, als Paradigmen der freien Schönheit der Episteme des Rokoko entstammen, wurzelt die Bestimmung der menschlichen Gestalt als Ideal der Schönheit im Frühklassizismus und verleiht dessen Emphase des antikisierenden nackten Körpers ein transzendentales Fundament. Man kann sich des Eindrucks nicht erwehren, dass hier jemand den Kopf vom Pulte hebt und aus dem Fenster blickt, auf die geschwungenen und geraden Linien eines Lustgartens, oder die vom Lesen ermüdeten Augen über das Muster der Tapete schweifen lässt, die das Raumgehäuse, in dem er sitzt, bekleidet, bevor sein Blick an den Schnörkeln eines Schranks zu Boden gleitet, oder in einer botanischen Monographie oder einem zoologischen Kompendium blättert oder von Briten verfasste, mit Stichen versehene Berichte ihrer Missionstätigkeit im Pazifik studiert, zum Beispiel die Natürliche und bürgerliche Beschreibung der Insel Sumatra in Ostindien (Leipzig 1785) von William Marsden. ${ }^{67}$

Auf solche oder ähnliche Art ist Kant wohl auch auf die "Neuseeländer" - gemeint sind freilich die Maori - und ihre Tätowierungen gestoßen und hat sich von den Zierraten in ihren Gesichtern zu der eingangs wiedergegebenen Reflexion über die Schönheit anregen lassen: Man würde »eine Gestalt mit allerlei Schnörkeln und leichten, doch regelmäßigen Zügen, wie die Neuseeländer mit ihrem Tätowieren tun, verschönern können, wenn es nur nicht ein Mensch wäre «. ${ }^{68}$ Die Konzessivkonstruktion in dieser Aussage lässt sich auf mehrfache Weise lesen. Man kann in ihr die historische Differenz zwi- 
schen Rokoko und Frühklassizismus erkennen, wie es vorliegender Aufsatz getan hat. Oder man kann die systematische Differenz zwischen freier und anhängender Schönheit in den Blick nehmen - zwischen der freien Schönheit der tätowierten Ornamente und der anhängenden Schönheit des Menschen als sittliches Wesen -, deren unterschiedliche Hinsichten auf ein und denselben Gegenstand Kant zufolge konfligierende Beurteilungen seiner Schönheit ermöglichen. ${ }^{69}$

Robert Wicks hingegen hat - mit und gegen Kant - argumentiert, dass die Platzierung tätowierter ornamentaler Formen auf einem menschlichen Gesicht auch im Rahmen der kantischen Philosophie keinen Widerspruch darstellen muss, wenn man den sichtbaren Ausdruck der Sittlichkeit des Menschen vom neoklassischen Ideal anatomischer Perfektion entkoppelt und ihn stattdessen formal als die grundlegende Möglichkeit künstlerischen Schaffens schlechthin fasst. ${ }^{70}$ Wicks zufolge bedeutet dies, eine teleologische Vorstellung der Schönheit des menschlichen Körpers in den Hintergrund zu drängen, die eine "ästhetische Normalidee " postuliert, welche - so Kant - »die Natur zum Urbilde ihren Erzeugungen in derselben Spezies unterlegte «? ${ }^{71}$ Dafür gelte es, die Naturhaftigkeit nicht nur im Werk des Genies, sondern in kreativer menschlicher Tätigkeit überhaupt zu betonen, wie beim Tätowieren von Gesichtslinien.

Schließlich lässt sich das Zitat auf die semantische Gegenüberstellung von »Neuseeländer« und "Mensch" hin befragen. Als Kant 1789 an der Kritik der Urteilskraft schrieb, lag die Entdeckung der Nordinsel Neuseelands durch den Engländer James Cook gerade einmal zwanzig Jahre zurück. 1777 war Cook zu einer zweiten Neuseelandfahrt aufgebrochen, begleitet vom deutschen Gelehrten Georg Forster, mit dem Kant in der zweiten Hälfte der 1780er-Jahre einen Disput über den Status des Begriffs der menschlichen $>$ Rasse $\triangleleft$, seine Unterteilungen und deren teleologische Implikationen führen sollte. ${ }^{72}$
Pauline Kleingeld hat nachgewiesen, dass das Konzept menschlicher `Rassen`, das Kant unter anderem in den Vorlesungen über Anthropologie und in den Vorlesungen über Physische Geographie entfaltete, für den Philosophen primär in naturgeschichtlicher Hinsicht interessant war. Er verwendete es, um die Diversität der physischen Erscheinungsformen unterschiedlicher Völker und deren relative historische Konstanz zu erklären. Zugleich aber, so Kleingeld, waren damit weitreichende völkerrechtliche und politische Annahmen verbunden: Diese unterstellten einerseits eine Hierarchie der `Rassen $`$ - mit den Europäern an der Spitze - und leiteten aus jener Rangordnung menschlicher >Rassen seits eine Legitimation des Status quo europäischer Kolonisierungen und - schlimmer noch von Sklaverei und Sklavenwirtschaft ab. ${ }^{73}$

Kleingeld konnte zeigen, dass in Arbeiten wie den Vorlesungen über Physische Geographie von 1782 die kantischen Semantiken von "Mensch" und »Neger« in ein Verhältnis harschen Gegensatzes treten, welches die Einheit des Gattungsbegriffs >Mensch ‘ aufs Äußerste anspannt. ${ }^{74}$ Parallel dazu bildete das Modell des Menschen als eines sittlichen Wesens, das sich seine Zwecke selbst zu setzen vermag, und des »Weltbürgerlichen " - soweit dieser Begriff in den kantischen Schriften vor 1795 auftaucht wie in der Idee zu einer allgemeinen Geschichte in weltbürgerlicher Absicht (1784) - für Kant eindeutig der europäische Mensch. Ihm wurde teleologisch die Aufgabe zugewiesen, die übrigen Völker zu Sittlichkeit und bürgerlicher Verfassung zu führen, ihnen »Gesetze zu geben«.75 Ab 1795 ändert sich mit der Schrift Zum ewigen Frieden und der Metaphysik der Sitten von 1797 Kants Auffassung über die Legitimität von Sklaverei und Kolonisierung radikal, ja sie kehrt sich ins genaue Gegenteil. ${ }^{76}$ Kolonisierungen, auch von Völkern, die selbst keine staatlichen Strukturen ausgebildet haben, werden für illegitim erklärt, die Vorstellung einer Hierarchie menschlicher 'Rassen verschwindet, der systematische Widerspruch 
zwischen dem Gebot, Menschen niemals als bloße Zwecke zu gebrauchen, und Kants früherer Apologie der Versklavung außereuropäischer Völker löst sich auf. ${ }^{77}$

Die Kritik der Urteilskraft wurde 1790 in erster Auflage veröffentlicht, zu einer Zeit also, in der Kant nach Kleingeld seine alten Überzeugungen noch nicht revidiert hatte. Man würde, schrieb der Philosoph, »eine Gestalt mit allerlei Schnörkeln und leichten, doch regelmäßigen Zügen, wie die Neuseeländer mit ihrem Tätowieren tun, verschönern können, wenn es nur nicht ein Mensch wäre $~_{7^{8}}$ Es ist zu vermuten, dass Kant den »Neuseeländern« um 1790 eine Stellung analog zu den »Indianern« Amerikas einräumte, ${ }^{79}$ die er durch eine gewisse Antriebslosigkeit und Unzivilisierbarkeit, ja die Unfähigkeit, sich selbst Zwecke zu setzen, kennzeichnete. ${ }^{80}$ Der Satz aus der Kritik der Urteilskraft lässt eine mit dieser Annahme konsistente Interpretation zu: Während die ungesitteten »Neuseeländer" Tätowierungen in menschlichen Gesichtern für schön halten mögen, verbietet das transzendentale Ideal der Schönheit, das von Kant gleichwohl nach europäischem, frühklassizistischem Muster gedacht wird, ein Anbringen solcher Verzierungen an der menschlichen Gestalt. Man mag einwenden, dass die emphatische Nennung des "Menschen" in vorliegender Passage als übergeordneter Gattungsbegriff auch auf die »Neuseeländer» bezogen werden müsse, dass es an sich bemerkenswert sei, dass die "Schnörkel und leichten, doch regelmäßigen Züge« in den Gesichtern ranghoher Maori als unstrittige Exempel freier Schönheit fungieren, dass sich hier im Ästhetischen vielleicht sogar ein Vorschein der kantischen Schriften nach 1795 ankündige. Es darf jedoch nicht übersehen werden, dass Kant die ornamentalen Tätowierungen der Maori nicht als Ergebnis kunstfertiger menschlicher Arbeit betrachtete, sie von ihrem rituellen, sozialen und symbolischen Bedeutungsgefüge in der MaoriKultur isolierte und als freie Schönheiten implizit in den Bereich der Natur rückte, so wie er die »Neuseeländer« noch 1793 als Beispiele von Menschen im »sogenannten Naturzustande« anführte, der nebenbei bemerkt als äußerst grausam vorgestellt wurde. ${ }^{81}$

XI.

Samuel E. Stuart war ebenfalls "Neuseeländer«, wenn auch keiner von jenen, die Kant im Sinne hatte, als er die vorstehenden Zeilen schrieb. Ansässig in Kingsland in der Nähe von Auckland, Künstler und Sekretär der Auckland Society of Arts, hatte er sich auf graphische und malerische Darstellungen der neuseeländischen Wildnis spezialisiert, die er häufig nach photographischen Motiven zu fertigen pflegte. Eine seiner Tuschezeichnungen, wohl um das Jahr 1890 entstanden, die ihrerseits nur als photographische Reproduktion überlebt hat, zeigt den Maori-Häuptling Tamati Waka Nene, geboren 1785 auf der Nordinsel Neuseelands - fünf Jahre vor Erscheinen der Kritik der Urteilskraft -, 1839 zu Jesus Christus bekehrt und auf den Namen »Thomas Walker» («Tamati Waka») getauft, gestorben 1871 (Abb. 3). Tamati Waka Nene war einer der wichtigsten Verbündeten der Briten im Zuge der Eroberung und Kolonisierung der neuseeländischen Inseln und ihrer Verteidigung gegen die Franzosen. Auf der Zeichnung, entstanden wohl einige Jahre nach Nenes Tod und auch sie vermutlich auf einer photographischen Vorlage basierend, sieht man diesen in traditionellem Ornat. Gesicht und Haltung drücken Ruhe, Würde und Gelassenheit aus. Die auf charakteristische Art in die Haut geritzten und mit dunklen Farbpigmenten versehenen labyrinthisch geschwungenen Ornamente oder $t \bar{a}$ moko sind gut zu erkennen. Sie kontrastieren in reizvoller Weise mit den kleinteiligeren Locken des Häuptlings und den strengen geometrischen Winkeln seines Gewandes. Stuarts Graphik beraubt die tā moko jedoch ihrer typischen dem Vernarbungsprozess geschuldeten Plastizität. Sie 


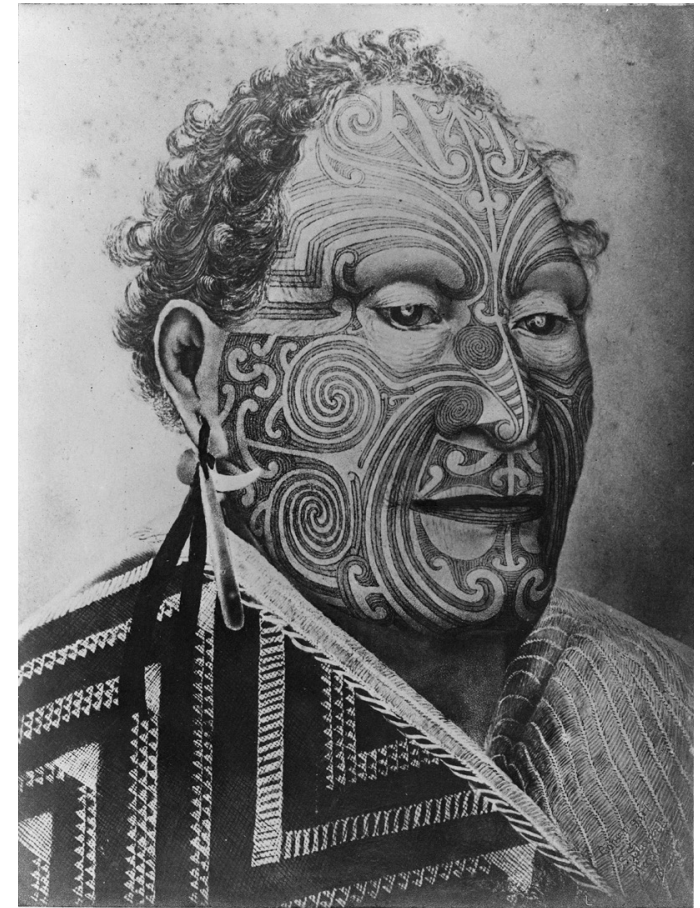

3 Samuel E. Stuart, Tamati Waka Nene alias Thomas Walker, hochrangiger Maori mit typischen Tätowierungen (tā moko), Federzeichnung, photographiert von John Reginald Wall, ca. 1890, Original verschollen. Wellington, National Library of New Zealand

erscheinen abstrakt und verflacht, als handele es sich lediglich um aufgezeichnete Lineamente, dem Gesicht des Häuptlings äußerlich, bloßes Beiwerk, das jederzeit und ohne Spuren wieder entfernt werden kann, um den `Menschen ' hinter der Maskerade des Wilden hervortreten zu lassen. Im Medium der Zeichnung wird Nene, mag er auch als Häuptling dargestellt sein, ‘zivilisiert.

Eine Photographie von 1870 zeigt ebenfalls Nene in seinen späten Jahren - hier jedoch als »Thomas Walker" (Abb. 4). Die Ritzungen in seinem Gesicht treten deutlich hervor, zugleich aber ist er europäisch gekleidet, trägt Anzug, Hemd und Halsbinde, ein vornehmer Bourgeois des 19. Jahrhunderts, der in jedem Pariser oder Londoner Salon hätte bestehen können - wären

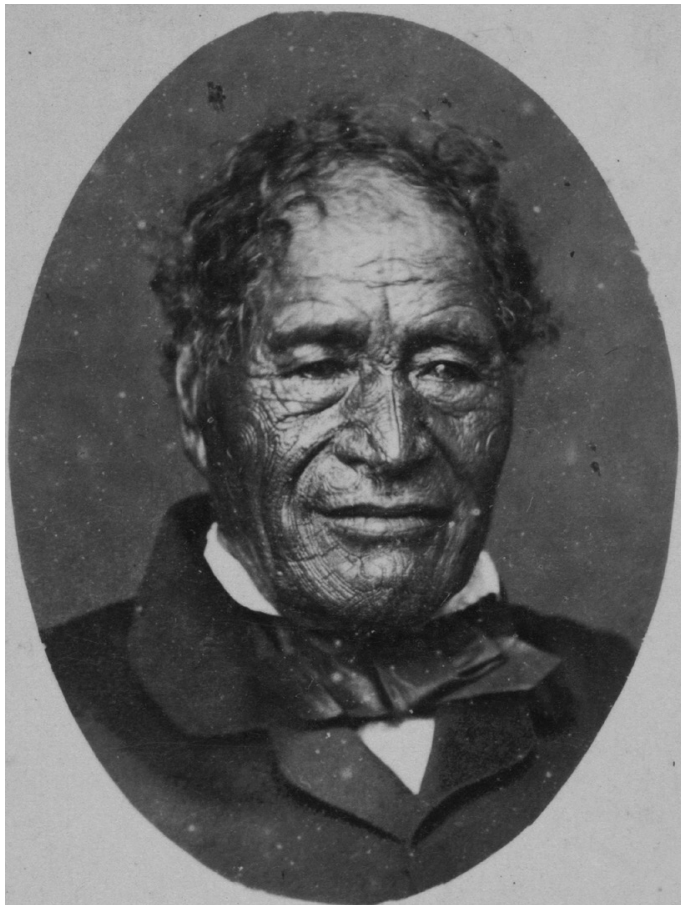

4 Hartley Webster, Portrait of Tamati Waka Nene, ca. 1870, Abzug auf Albuminpapier. Wellington, National Library of New Zealand

da nicht die Tätowierungen, die seiner Kleidung etwas Unwirkliches, Kostümiertes und Pastichehaftes verleihen. Sind die "Zieraten" in Nenes Gesicht als schön im Sinne des kantischen Geschmacksurteils anzusprechen? Wir erinnern uns, eine der historischen Bedeutungen von "Zieraten" war eben auch "Tätowierungen". Sie sind es, und sie sind es nicht. Als ornamentale Formen sind die Tätowierungen »Muster«, $\mathrm{Pa}$ radigmen der freien, nicht an bestimmte Objekte gebundenen Schönheit. Als solche sind sie Gegenstände des ästhetischen Urteils par excellence. Andererseits befinden sie sich eingeritzt im Gesicht eines Menschen - und dort können sie Kant zufolge nicht schön sein, allen Idealisierungsbemühungen in Stuarts Zeichnung zum Trotz. 


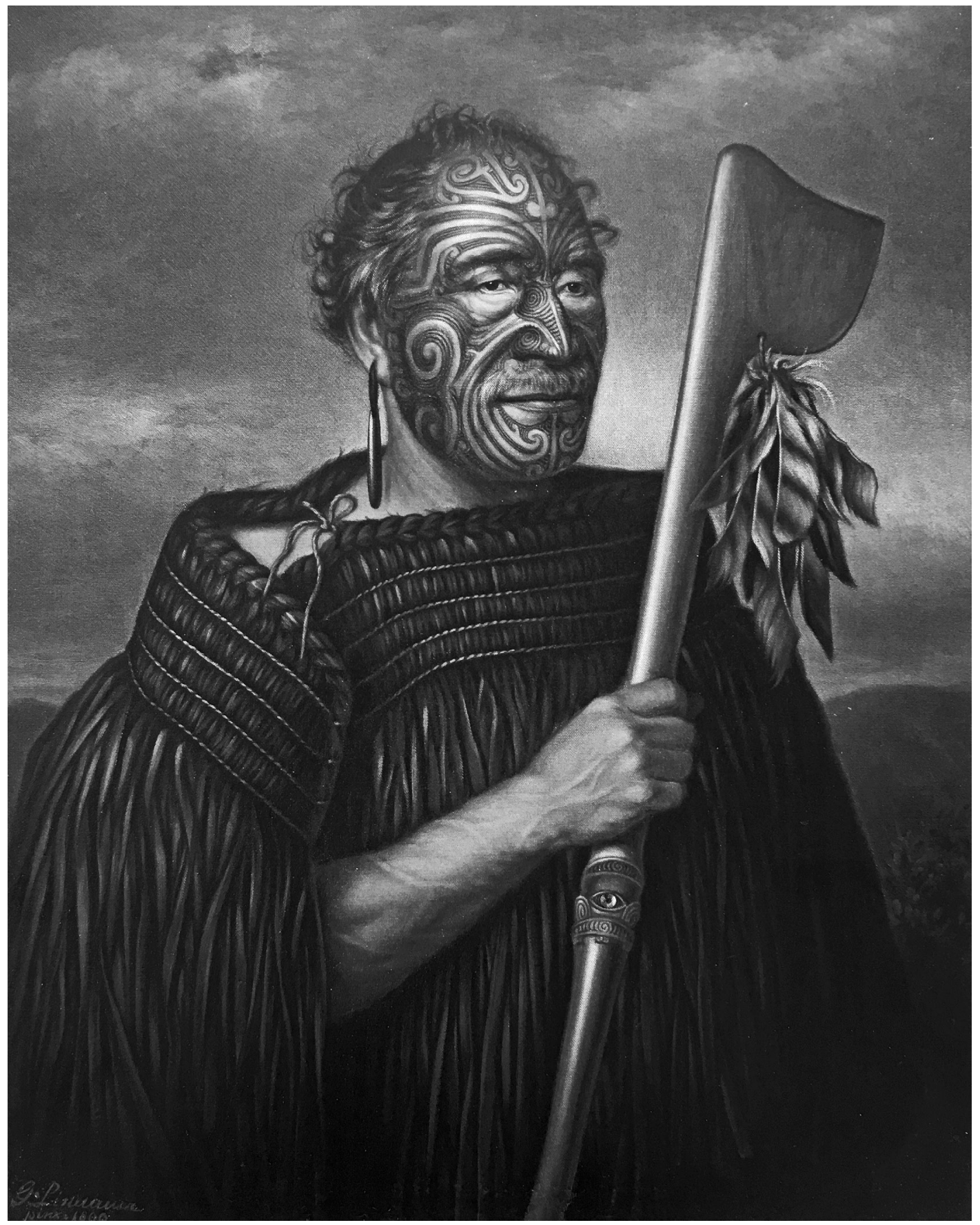

5 Gottfried Lindauer, Tamati Waka Nene, 1890, Öl auf Leinwand, 88 x 70 cm. Auckland Art Gallery Toi o Tāmaki 
Die $t a \bar{a}$ moko lassen das Ideal der Schönheit, die menschliche Gestalt, insofern diese sichtbarer Ausdruck der Idee des Sittlichen ist, als MaoriHäuptling in Erscheinung treten. Das Ideal der Schönheit wird so zur "ästhetischen Normalidee« herabgesetzt, die eben nur für bestimmte Völker und für bestimmte Breiten gilt. In Gestalt der Normalidee generiert die Einbildungskraft Kant zufolge eine Art Durchschnittsbild des Gattungswesens Mensch, das als notwendiger Maßstab der »Richtigkeit», keineswegs aber als hinreichende Bedingung menschlicher Schönheit dient: »Wenn nun [...] für diesen mittleren Mann der mittlere Kopf, für diesen die mittlere Nase usw. gesucht wird, so liegt diese Gestalt der Normalidee des schönen Mannes in dem Lande, wo diese Vergleichung angestellt wird, zum Grunde; daher ein Neger notwendig unter diesen empirischen Bedingungen eine andere Normalidee der Schönheit der Gestalt haben muß, als ein Weißer, der Chinese eine andere als der Europäer. Mit dem Muster eines schönen Pferdes oder Hundes (von gewisser Rasse) würde es ebenso gehen ${ }^{82}{ }^{82}$

Es sind jedoch europäische Muster im Geist des Frühklassizismus - für die anhängende Schönheit der idealen menschlichen Gestalt und des Rokoko - für die freie Schönheit -, die Kant im Auge hat, wenn er von der »Einhelligkeit, soviel als möglich, aller Zeiten und Völker« in Fragen des Geschmacks handelt. Umso irritierender muten Anzug, Hemd und Halsbinde auf der Photographie Tamati Waka Nenes an. Sie scheinen dem gekerbten, tätowierten Gesicht des Häuptlings einen "weltbürgerlichen", und das heißt für Kant vor 1795 einen europäischen Rahmen zu verleihen, der die Ornamente nicht nur von ihrem lokalen Bezugssystem in der MaoriKultur der Nordinsel Neuseelands absondert, sondern auch und gerade ihre ästhetische Funktion selbst auslöscht - zugunsten eines weltbürgerlichen Bewusstseins, dem die Zeichen in Nenes Gesicht nicht anders erscheinen können denn als Narben. Doch dieser Rahmen, die Kleidung eines Dandys oder Bourgeois, ist selbst der Zeit und der Geschichte unterworfen. Er ist selbst nur ein Beiwerk und nicht das sichtbare Anzeichen eines zivilisierten Weltbürgertums. Wir können heute nicht umhin, in ihm die Staffage und Maskerade des 19. Jahrhunderts zu erblicken. ${ }^{83}$

Auch der ungleich bekanntere Zeitgenosse Stuarts, der aus Böhmen nach Neuseeland eingewanderte Maler Gottfried Lindauer, der zwischen der Mitte der 1870er-Jahre und seinem Tod 1926 dutzende Porträts von ranghohen Maori beiderlei Geschlechts fertigte, hat Nene porträtiert (Abb. 5). ${ }^{84}$ Die Leinwand wurde 1890 - Nene war bereits 19 Jahre verschieden - nach einer Carte de Visite-Aufnahme der Aucklander Photographin Elizabeth Pulman gemalt, eine für Lindauer durchaus übliche Praxis, der seine Maori-Bildnisse sowohl nach photographischen Reproduktionen als auch nach dem lebenden Modell schuf. In Lindauers typischem, monumentalisierendem Stil zeigt das Porträt Nene in halbfiguriger Darstellung, den Kopf nach rechts gewandt. Er trägt ein traditionelles pākē oder Regencape. Seine Rechte hält mit entschlossenem Griff eine kunstvoll geschnitzte, mit Federn geschmückte Stockwaffe, in die eine glänzende Muschelschale in Form eines Auges eingelegt ist. Lindauer hat Nene vor einem leicht bewölkten Himmel und einer unbestimmten Hügellandschaft in der Dämmerung dargestellt. Das Porträt drückt eine beinahe antike Gelassenheit, Kraft und Würde aus. Es bildet hier keine Ausnahme: Die gesamte Reihe von Lindauers MaoriBildern mutet durch ihren klassizistischen, antikisierenden Duktus und die präzise, im späten 19. Jahrhundert bereits antiquierte Feinmalerei wie eine Galerie römischer Bürger an.

Die von Lindauer wiedergegebenen spiralförmigen Tätowierungen in Nenes Gesicht gleichen den Lineamenten auf Stuarts Zeichnung bis ins Detail, von der Andeutung eines weißen Oberlippenbartes bei Lindauer abgesehen, welcher den Häuptling älter wirken lässt. Auch die Darstellung des Kopfes im Dreiviertelprofil ist identisch. 
Es kann vermutet werden, dass dieselbe Carte de Visite beiden Künstlern als Vorlage diente oder dass Stuart sein Brustbild Nenes gar nach dem Vorbild von Lindauers Gemälde zeichnete. Beide Werke entstanden etwa zur selben Zeit, und beide Maler hatten enge biographische Bezüge zu Auckland. Darüber hinaus weiß man aufgrund einer zeitgenössischen photographischen Reproduktion, dass Stuart noch im August 1904 ein Maori-Porträt im Stil Lindauers malte und selbst die Form der Signatur an jene Lindauers anglich.

Ein wichtiger Unterschied der beiden Darstellungen besteht in der Kleidung Nenes. Während er bei Lindauer ein $p \bar{a} k \bar{e}$ trägt, ist er bei Stuart in einen kaitaka gehüllt, einen fein gewebten Umhang mit ornamentierter Borte. Geht man davon aus, dass beide Werke unmittelbar oder mittelbar auf dieselbe Photographie zurückgehen, muss man annehmen, dass sich die Künstler gewisse Freiheiten bei deren Umsetzung nahmen. Es war ein gewohntes Prozedere Lindauers, wenn er nach Photographien arbeitete, die Maori-Modelle in den gemalten Bildern nach eigenen Vorstellungen auszustaffieren, ihnen Ohrringe, Waffen und Schmuck zu verleihen, wie es ihm passend erschien, die Hintergründe zu wählen, die Porträtierten älter oder jünger zu machen, die tā moko leicht zu adaptieren. Man weiß, dass der Künstler über eine Sammlung maorischer `Requisiten` verfügte. Er benutzte sie nicht anders, als es die Photographen seiner Zeit in ihren Ateliers taten.

Am schlagendsten bleibt jedoch die Differenz des Gewandes. Viele ranghohe Maori wie Nene trugen ihre europäisch-bürgerliche Tracht auf den Carte de Visite-Photographien mit Stolz. Der Pinsel Lindauers versetzte sie zurück in einen idealisierten ikonographischen $>$ Naturzustand und stilisierte sie zugleich - der spätklassizistischen Form der Gemälde nach - zu >Weltbürgern als sittliches Wesen. Hundert Jahre nach dem Erscheinen der Kritik der Urteilskraft artikuliert sich der Gegensatz von freier und anhängender Schönheit in Lindauers Bildnis Tamati Waka Nenes in einer spezifischen Dialektik von menschlicher Gestalt und schmückendem Beiwerk: Die ideale, klassische Gestalt des Menschen, die seine transzendentale Sittlichkeit ausdrückt, erscheint als das, was bei allem Wechsel des Beiwerks unveränderlich bestehen bleibt. Aber die tätowierten, geritzten Linien der tā moko? Gehören sie zur Gestalt oder zum Beiwerk? Während die kantische Antwort klar ist, liegt der Fall bei Lindauer komplizierter. Trotz gelegentlicher Anpassungen von Details wie der Abdunkelung des Farbtons waren ihm die Ornamente in den Gesichtern der Maori nichts, was weggelassen oder beliebig geändert werden könnte. Er stellte sie vielmehr in unauflöslicher Verbindung mit den Gesichtern dar, als Formen, die das menschliche Antlitz nicht zum Normaltypus spezifizieren, sondern seinen Ausdruck steigern. In diesem gesteigerten Ausdruck verbindet sich die kantische intelligible Dimension der Würde des Menschen mit einer historischen, zutiefst melancholischen Dimension.
1 Steven Adams, Review: James Trilling, Ornament. A modern perspective \& David Brett, Rethinking decoration. Pleasure and ideology in the visual arts, in: Journal of Design History 19, 2006, 88 - 90, hier 89.

2 Ebenda.

3 Rudolf Arnheim, Review: Oleg Grabar, The meditation of ornament, in: The Journal of Aesthetics and Art Criticism 53, 1995, 218-219, hier 218 (Hervorhebungen des Verfassers).
4 Oleg Grabar, The mediation of ornament, Princeton 1992, 284; zit. nach Arnheim 1995 (wie Anm. 3), 218.

5 Vgl. Jacques Derrida, Die Wahrheit in der Malerei, hg. von Peter Engelmann, Wien 1992, 465.

6 Vgl. Immanuel Kant, Kritik der Urteilskraft, hg. von Heiner F. Klemme, Hamburg 2006, 83-86 [B 48-B 52]. Hier und im Folgenden verweist der Klammerausdruck auf die Originalpaginierung der zweiten, verbesserten Auflage der Kritik der Urteilskraft, die im 
Oktober/November 1792 (offizielles Druckjahr 1793) erschien und auf welche die von Heiner F. Klemme herausgegebene Ausgabe des Meiner-Verlags mit $» \mathrm{~B}[$ Seitenzahl] « Bezug nimmt.

7 Ebenda, 84 [B 49].

8 Ebenda, 102 [B 71].

9 Ebenda.

10 Ebenda, 102 [B 72].

11 Vgl. ebenda, 100 [B 70].

12 Ebenda, 103 [B 72].

13 Ebenda, 84-85 [B 50].

14 Vgl. u.a. Gayatri Chakravorty Spivak, A critique of postcolonial reason. Toward a history of the vanishing present, Cambridge, Mass. 1999, 449.

15 Kant 2006 (wie Anm. 6), 35 [B XLVII].

16 Vgl. Derrida 1992 (wie Anm. 5), 91 - 98.

17 Kant 2006 (wie Anm. 6), 19 [B XXVI].

18 Ebenda, 33 [B XLIV].

19 Ebenda, 173 [B 155].

20 Ebenda, 80 [B 45].

21 Ebenda, 80 [B 45-B 46].

22 Vgl. ebenda $21-28$ [B XXIX - B XXXVIII].

23 Vgl. David Morgan, The idea of abstraction in German theories of the ornament from Kant to Kandinsky, in: The Journal of Aesthetics and Art Criticism 50, 1992, $231-242$, hier 232.

24 Vgl. Kant 2006 (wie Anm. 6), 77-78. [B 42-B 43].

25 Vgl. ebenda, 78 [B 42-B 43].

26 Ebenda, $78-79$. [B 43].

27 Ebenda, 79 [B 43].

28 Vgl. Morgan 1992 (wie Anm. 23), 231-232; vgl. auch Helmut Pfotenhauer, Klassizismus und Ornament. Die italienischen Verzierungen in der deutschen Kunstdiskussion des 18. Jahrhunderts, in: FrankRutger Hausmann (Hg.), »Italien in Germanien«. Deutsche Italien-Rezeption von 1750-1850, Akten des Symposiums der Stiftung Weimarer Klassik Herzogin Anna Amalia Bibliothek, Tübingen 1996, $37-63$, hier $56-61$.

29 Vgl. Goethe-Wörterbuch, hg. von der Berlin-Brandenburgischen Akademie der Wissenschaften, der Akademie der Wissenschaften in Göttingen und der Heidelberger Akademie der Wissenschaften, Stuttgart 1978ff., URL: http://www.woerterbuchnetz.de/GWB? lemma=beiwerk (letzter Zugriff am 27. August 2017).

$30 \mathrm{Vgl}$. Deutsches Wörterbuch von Jacob und Wilhelm Grimm, Leipzig 1854-1961, URL: http://www. woerterbuchnetz.de/DWB?lemma=beiwerk (letzter Zugriff am 27. August 2017).

31 Kant 2006 (wie Anm. 6), 80 [B 44].

32 Ebenda, 100 [B 70].

33 Ebenda.

34 Ebenda, 83 [B 48].

35 Ebenda, 83 [B 49].

36 Ebenda.

37 Ebenda, 83-84 [B 49].
38 »Aber wo nur ein freies Spiel der Vorstellungskräfte [...] unterhalten werden soll, in Lustgärten, Stubenverzierung, allerlei geschmackvollem Geräte u. dergl., wird die Regelmäßigkeit, die sich als Zwang ankündigt, soviel möglich vermieden; daher der englische Geschmack in Gärten, der Barockgeschmack an Möbeln die Freiheit der Einbildungskraft wohl eher bis zur Annäherung zum Grotesken treibt, und in dieser Absonderung von allem Zwange der Regel eben den Fall setzt, wo der Geschmack in Entwürfen der Einbildungskraft seine größte Vollkommenheit zeigen kann«. Ebenda, 102 [B 71-B 72]; vgl. auch ebenda, $102-104$ [B 71-B 73].

39 Hier und im Folgenden vgl. Frank-Lothar Kroll, Zur Problematik des Ornaments im 18. Jahrhundert, in: Ursula Franke und Heinz Paetzold (Hg.), Ornament und Geschichte. Studien zum Strukturwandel des Ornaments in der Moderne (Zeitschrift für Ästhetik und allgemeine Kunstwissenschaft, Beiheft 2), Bonn 1996, $63-87$.

40 Vgl. Kant 2006 (wie Anm. 6), 102 [B 71-B 72].

41 Vgl. Pfotenhauer 1996 (wie Anm. 28), 47-50.

42 Ebenda, 59.

43 Kant 2006 (wie Anm. 6), 78 [B 43].

44 Ebenda, 79 [B 43].

45 Ebenda, 162 [B 142].

46 Ebenda, 503-504 (Hervorhebung des Verfassers).

47 Ebenda, 94 [B 63] (Hervorhebungen des Verfassers).

48 Ebenda, 94 [B 62-B 63].

49 Ebenda, 173 [B 155] (Hervorhebung des Verfassers).

50 Ebenda, 87 [B 53] (Hervorhebungen des Verfassers).

51 Vgl. ebenda, 87-88 [B 53-B 54]. Zu Kants Zeiten hatte >Muster - etymologisch eigentlich etwas, das 'gezeigt « wird - noch nicht wie heute alltagssprachlich die Bedeutung einer ornamentalen Flächengestaltung, etwa in dem Ausdruck sein gemustertes Kleid . >Muster bedeutete zeitgenössisch ausschließlich `Vorlage`, >Vorbild`, >Probestück`. Die Muster oder Vorbilder des »durch Beispiele bewährten Geschmacks « in der Kritik der Urteilskraft sind jedoch häufig Muster im Sinne von Ornamenten.

52 Ebenda, 87, Fußnote 1 [B 54].

53 Vgl. ebenda, 174 [B 157].

54 Vgl. ebenda, 174-175 [B 157-B 158].

55 Vgl. ebenda, 86-93 [B 53-B 61].

56 Ebenda, 88 [B 54].

57 Ebenda, 92 [B 60].

58 Ebenda, 84 [B 50].

59 Vgl. Gérard Raulet, Ornament und Geschichte. Strukturwandel der repräsentativen Öffentlichkeit und Statuswandel des Ornaments in der Ästhetik des 18. Jahrhunderts, in: Franke und Paetzold 1996 (wie Anm. 39), 19-43, hier 31-32.

60 Vgl. Kant 2006 (wie Anm. 6), 85 [B 50].

61 Kant unterscheidet "Natur" in formaler und in materieller Hinsicht. In formaler Hinsicht ist 
Natur »Natur überhaupt«, das heißt eine Gesamtheit von Erscheinungen, insofern sie unter Gesetzen des Verstandes stehen. Vgl. Immanuel Kant, Kritik der reinen Vernunft, hg. von Raymund Schmidt, Hamburg 1990, 183-186 [B 163-165]. Diese Gesetze, etwa das Kausalgesetz, sind transzendentaler Art. Sie lassen sich a priori angeben und werden in der Kritik der reinen Vernunft untersucht. Im Bereich der Natur überhaupt gibt es keine Überraschungen. Die Kritikder Urteilskraft hingegen beschäftigt sich mit der Natur in materieller Hinsicht, mit der Natur als »dem Inbegriffe aller Erscheinungen«. Ebenda, 183 [B 163]. »Natur« wird hier zum Synonym der empirischen Welt in der unausrechenbaren Vielfalt ihrer spezifischen Gesetze und Gestalten. "Allein es sind so mannigfaltige Formen der Natur, gleichsam so viele Modifikationen der allgemeinen transzendentalen Naturbegriffe, die durch jene Gesetze, welche der reine Verstand a priori gibt, [...] unbestimmt gelassen werden, daß dafür doch auch Gesetze sein müssen die zwar, als empirische, nach unserer Verstandeseinsicht zufällig sein mögen, die aber doch [...] aus einem, wenn gleich uns unbekannten, Prinzip der Einheit des Mannigfaltigen als notwendig angesehen werden müssen«. Kant 2006 (wie Anm. 6), 19 [B XXVI]. Ein solches unbekanntes Prinzip aber kann niemals transzendentalen Charakter haben. Es kann nur reflektierend, im Modus des `als ob<, unterstellt werden. Vorliegender Text fasst `Natur durchgehend in materieller Hinsicht, das heißt als empirische Natur.

62 Ebenda, 99 [B 69].

63 Ebenda, 107 [B 77].

64 Ebenda, 108 [B 77].

65 Ebenda, 192 [B 180].

66 Richard Heinrich, Schweres Luftgewebe. Kant und das Rokoko, in: Herbert Lachmayer (Hg.), Mozart. Experiment Aufklärung im Wien des ausgehenden 18. Jahrhunderts, Ostfildern 2006,75-85, hier 8o-81. Mit der Muschelform der Rocaille sind freilich auch jene "Schaltiere des Meeres« angetönt, die Kant scheinbar im Vorübergehen in $\$ 16$ erwähnt. Zum unterschwelligen Rokoko-Bezug in Kants Ästhetik vgl. auch Gernot Böhme, Kants 'Kritik der Urteilskraft in neuer Sicht, Frankfurt a. M. 1999, 12-40. Anders als Heinrich, der das Rokoko in der Tradition Michel Foucaults als eine epistemologische Formation mit bestimmten Strukturmerkmalen denkt, fasst Böhme das Rokoko in erster Linie als Stilepoche: »Kant ist ein Mensch des Rokoko« (26). Entsprechend begegnen und überkreuzen sich Kunst und Natur bei Böhme im Salon. Kant selbst habe weder ausgiebige
Kunst- noch Naturerfahrungen gemacht. »Im ganzen verdichtet sich der Eindruck, daß die Beispiele, an denen Kant seinen Begriff des Schönen ausbildete, weder der freien Natur noch der autonomen Kunst angehören, sondern vielmehr dem lebensweltlichen Bereich, in dem man durch Dekor, Verzierungen, Arrangements, `Putz« Verschönerung betreibt« (22). Über dem Rokokokontext dürfen die Bezüge der kantischen Ästhetik zum Frühklassizismus jedoch keinesfalls vergessen werden. Vgl. dazu Pfotenhauer 1996 (wie Anm. 28); Kroll 1996 (wie Anm. 39).

67 Vgl. Kant 2006 (wie Anm. 6), 443 - 444.

68 Ebenda, 84-85. [B 50].

69 Vgl. ebenda, 84-86 [B 50-B 52].

$70 \mathrm{Vgl}$. Robert Wicks, Kant on beautifying the human body, in: British Journal of Aesthetics 39, 1999, 163-178. Wicks weist zu Recht darauf hin, dass die spiralförmigen Lineamente der Gesichtstätowierungen der Maori der snatürlichen` Topographie und plastischen Organisation des menschlichen Gesichts nicht zuwiderlaufen, sondern diese im Gegenteil unterstreichen.

71 Vgl. Kant 2006 (wie Anm. 6), 91.

72 Vgl. Katrin Flikschuh und Lea Ypi, Introduction: Kant on colonialism - apologist or critic?, in: dies. (Hg.), Kant and colonialism. Historical and critical perspectives, Oxford 2014, 1-18, hier 5-6; vgl. auch im selben Band: Pauline Kleingeld, Kant's second thoughts on colonialism, 43-67, hier 49-50.

73 Vgl. ebenda, 43-52.

74 Vgl. ebenda, 47.

75 Vgl. ebenda, 43-45.

$76 \mathrm{Zu}$ den möglichen Gründen für diesen Umschwung vgl. ebenda, 64-65.

77 Vgl. ebenda, 54.

78 Kant 2006 (wie Anm. 6), 84-85. [B 50].

79 Vgl. Kleingeld 2014 (wie Anm. 72), 51 und 54; vgl. auch Immanuel Kant, Die Religion innerhalb der Grenzen der bloßen Vernunft, in: ders., Akademieausgabe von Immanuel Kants Gesammelten Werken, Bd. 6, Berlin/ New York 1977, 1-202, hier 33.

8o Vgl. Kleingeld 2014 (wie Anm. 72), 47 und 51.

81 Vgl. Kant 1977 (wie Anm. 79), 33.

82 Kant 2006 (wie Anm. 6), 91 [B 58].

83 Zum Problem der Kleidung auf Photographien vergangener Epochen als Staffage vgl. Siegfried Kracauer, Die Photographie, in: ders., Das Ornament der Masse, Frankfurt am Main 1977, 21-39.

84 Vgl. Gottfried Lindauer. Die Maori Porträts (Ausst.Kat. Berlin, Alte Nationalgalerie), hg. von Udo Kittelmann und Britta Schmitz, Köln 2014, 74.

Abbildungsnachweis: 1 M. Klammer. — 2 National Gallery of Australia, Canberra, Inv. NGA 2007.1774.38.1. — 3 National Library of New Zealand, Wellington, Inv. 1/1-017878-F. — 4 National Library of New Zealand, Wellington, Inv. PA2-2211. — 5 Ausst.-Kat. Gottfried Lindauer 2014 (wie Anm. 84), 75. 